\title{
Identification of prognostic biomarkers in colorectal cancer using a long non-coding RNA-mediated competitive endogenous RNA network
}

\author{
MINJIE HE ${ }^{1 *}$ YAN LIN ${ }^{2 *}$ and YUZHEN XU ${ }^{3}$ \\ ${ }^{1}$ Department of Medical Oncology, The First Affiliated Hospital of Kunming Medical University, Kunming, \\ Yunnan 650000; ${ }^{2}$ Department of Oncology, The Affiliated Traditional Chinese Medical Hospital of \\ Xinjiang Medical University, Urumqi, Xinjiang 830000; ${ }^{3}$ Department of Gastrointestinal Surgery, \\ Xuzhou Hospital Affiliated to Medical School of Southeast University, Xuzhou, Jiangsu 221009, P.R. China
}

Received May 28, 2018; Accepted December 5, 2018

DOI: $10.3892 / \mathrm{ol} .2019 .9936$

\begin{abstract}
Colorectal cancer (CRC) is a highly malignant gastrointestinal tumor accompanied by poor prognosis. Long non-coding RNA (lncRNA) plays an important role in the progression and physiology of tumors as it competes with endogenous RNAs, including miRNA and mRNA. In the present study, a multi-step computational method was used to build a CRC-related functional lncRNA-mediated competitive endogenous RNA (ceRNA) network (LMCN). 1ncRNAs with more degrees and betweenness centrality (BC) were screened out as hub lncRNAs. Then functional enrichment analyses of IncRNAs were carried out from the Gene Ontology (GO) and Reactome pathway databases based on the guilt by association' principle. As a result, lncRNAs in the LMCN displayed specific topological characteristics in accordance with the regulatory correlation of coding mRNAs in CRC pathology. HCP5, EPB41L4A-AS1, SNHG12, and LINC00649 were screened out as hub lncRNAs which were more significantly related to the development and prognosis of CRC. The hub lncRNAs in CRC were obviously involved in functions of cell cycle arrest, vacuolar transport, histone modification, and in pathways of GPCR, signaling by Rho GTPases, axon guidance pathways, meaning that they might be potential biomarkers for diagnosis, evaluation and gene-targeted therapy of CRC. Thus, the LMCN construction method could accelerate lncRNA discovery and therapeutic development in CRC.
\end{abstract}

Correspondence to: Dr Yuzhen Xu, Department of Gastrointestinal Surgery, Xuzhou Hospital Affiliated to Medical School of Southeast University, 199 Jiefang South Road, Quanshan, Xuzhou, Jiangsu 221009, P.R. China

E-mail: yuzhenxu12@126.com

${ }^{*}$ Contributed equally

Key words: ceRNA network, colorectal cancer, lncRNA, topology, pathogenesis, prognostic biomarker

\section{Introduction}

Colorectal cancer (CRC), usually starting as a harmless polyp, is one of the most common gastrointestinal malignancies. Globally, CRC is the third most common cancer in the diagnosis of male diseases, ranking second in women. Approximately 1.4 million new CRC cases and 700,000 CRC deaths were evaluated in 2012. It has been found that the incidence of CRC is highest in North America, Europe, and Australia/New Zealand, and other countries with lower history rate are currently facing increased risk (1). The risk factors of CRC include environment (such as obesity, alcohol consumption and tobacco smoking), heredity and gene-environment interactions (2). Stool tests, sigmoidoscopy or colonoscopy, and virtual colonoscopy are common clinical screening methods for CRC (3). Although the fecal occult blood testing and sigmoidoscopy can provide valuable information for the guide therapy of CRC, they are not sufficient to confirm clinical outcomes. Therefore, seeking new effective diagnostic methods and exploring the canceration mechanism from polyp becomes urgent. It has been reported that bioinformatics analysis of gene expression profiles could clearly explain the occurrence, development, metastasis and prognosis of tumors, and is a hot spot in the current basic and clinical research (4-6).

Long non-coding RNA (lncRNA) is a novel class of transcripts with 200 nucleotides that do not serve as a template for protein (7), but they are involved in a variety of biological processes, such as epigenetic regulation, chromosome remodeling and gene expression regulation (8). Studies have found that lncRNAs are abnormally expressed in many tumors, including non-small cell lung cancer, cervical cancer, hepatocellular cancer, and human breast cancer (9-12). The expression level of other transcripts as miRNA sponges could be regulated by these lncRNAs through competitive endogenous RNAs (ceRNAs) network $(13,14)$. Furthermore, some canonical oncogenic pathways can be revealed by ceRNA interaction network (15), and Wang et al have reported that the newly identified ceRNA network contributes to the exploration of the regulatory mechanisms of ceRNA mediated by lncRNA in the nosogenesis of muscle-invasive bladder cancer (16). 
However, it remains a challenge to identify lncRNA biomarkers of CRC, and to understand the functional roles of ceRNAs mediated by lncRNA in CRC.

In the present study, a functional lncRNA-mediated ceRNA network (LMCN) associated with CRC was constructed using a multi-step computational method, and the relevant lncRNAs were identified based on the constructed landscape map. Then we carried out functional enrichment analyses for mRNAs which were significantly associated with IncRNAs, and used the functions of the mature mRNAs to forecast the lncRNA functions.

\section{Materials and methods}

\section{Data source}

Expression profiles of $\ln c R N A$ and $m R N A$ in $C R C$. In the present study, the gene expression profile dataset no. GSE31737, deposited by Loo et al (17) in the Gene Expression Omnibus (GEO) database (http://www.ncbi.nlm.nih.gov/geo/) based on the GPL5175 platform [HuEx-1_0-st; Affymetrix Human Exon 1.0 ST Array: transcript (gene) version; Affymetrix; Thermo Fisher Scientific, Inc., Waltham, MA, USA], was subjected to bioinformatics analysis. A total of 80 chips were involved in the dataset, including 40 colon cancer tissues (tumor group) and 40 paired adjacent-normal colon tissues (control group). After preprocessing and mapping between genes and probes, we obtained 14,451 gene expression profile data.

Identification of miRNA-target interactions. Firstly, interactions between miRNA and mRNA, and between miRNA and lncRNA were obtained from the starBase v2.0 which provided the most comprehensive decoding of miRNA-ceRNA, miRNA-ncRNA and protein-RNA interaction networks from large-scale CLIP-Seq data (18). Then a new expression profile was acquired through taking the intersection between the genes in the above-mentioned expression profile data of CRC and the mRNAs and lncRNAs, respectively, which were located in the interactions of miRNA-mRNA and lncRNA-miRNA. Subsequently, the interaction relationship of the new gene expression profile were extracted from the downloaded interactions of miRNA-mRNA and miRNA-IncRNA.

Construction of LMCN. The competitive IncRNA-mRNA interactions were identified using a hypergeometric test that was able to effectively assess the significance of the miRNAs shared by each IncRNA and mRNA. There were a total of $N$ miRNAs in the genome, where $K$ and $M$ represented the number of miRNAs related to the current IncRNA and mRNA, and $x$ represented the number of common miRNAs shared by IncRNA and mRNA. P-value was calculated in order to evaluate the enrichment significance for that function using the formula:

$$
\mathrm{P}=1-\sum_{n=1}^{x} \frac{\left(\begin{array}{l}
K \\
t
\end{array}\right)\left(\begin{array}{l}
N-K \\
M-t
\end{array}\right)}{\left(\begin{array}{l}
N \\
M
\end{array}\right)}
$$

False discovery rate (FDR) correction was performed to adjust the P-values. FDR $<0.01$ was used as the threshold of LMCN.

The IncRNA-mRNA interaction-pairs screened out above were co-expression analyzed through calculating their
Pearson's correlation coefficient in the control and tumor group. The calculation formula used was:

$$
\rho_{X, Y}=\frac{\operatorname{cov}(\mathrm{X}, \mathrm{Y})}{\sigma_{X} \sigma_{Y}}
$$

of which $\operatorname{cov}(\mathrm{X}, \mathrm{Y})$ represents the covariance of variables $\mathrm{X}$ and $\mathrm{Y}$, and $\sigma_{X}$ and $\sigma_{Y}$ represent the standard deviations of $\mathrm{X}$ and $\mathrm{Y}$, respectively. FDR $<0.01$ was set as the threshold. The lncRNA-mRNA interaction-pairs whose difference values of Pearson's correlation coefficient was $>0.3$ between control and tumor groups were taken as the significant co-expression ceRNA interactions (19). The ceRNA network was constructed and described using a Cytoscape software (v3.5.1; Cytoscape Consortium, New York, NY, USA). A complete bipartite graph was involved in this model in which an edge was achieved from each apex of the lncRNA set to each vertex of the mRNA set.

We studied the relationship between the number of genes and the degree of distribution, and the fitting coefficient $\mathrm{R}^{2}$ of the power-law $\mathrm{Y}=\mathrm{aX}$ of the objective networks was detected (20). The built-in Network Analyzer tool (Agilent Technologies, Inc., Santa Clara, CA, USA) was used to analyze the node degree and the betweenness centrality (BC). $\mathrm{BC}$ represents a central metric for the nodes in the network. It can be obtained by computing the number of the shortest paths through the node, from each node to all others. This property reflects the control ability or the probability to exert the 'gate-keeping' effect of a node in the ceRNA network. Generally, a higher degree implies that the node is a hub involved in more ceRNA interactions. A higher BC indicates that the node is a bottleneck that functions as a bridge to connect different network modules.

Screening of hub lncRNAs. LMCN can provide an all-sided landscape of all possible ceRNA interactions, which contributes in exploring the regulatory roles of the lncRNAs. Moreover, more detailed view on the synergy between IncRNAs and competing mRNAs can be confirmed by analyzing the regional sub-networks. A high-competitive sub-network was extracted from the LMCN based on a Pearson's correlation coefficient threshold $>0.5$. IncRNAs with more degrees and BCs were selected out as hub lncRNAs involved in CRC.

Function prediction of IncRNAs. Functions of the hub IncRNAs can be studied with a 'guilt by association' (GBA) approach $(21,22)$. It has been stated by GBA principle that genes that participate in the same biological process are inclined to be correlated (or have similar properties, such as similar expression patterns), allowing statistically to infer previously unknown functions of a gene based on some previous knowledge on other genes and correlative data $(23,24)$. In this study, the GBA meant that the function of IncRNAs could be deduced though carrying out a functional enrichment analysis for the mature mRNAs which were significantly associated with lncRNAs.

Function and pathway enrichment analysis were performed to evaluate the lncRNAs competitive mediated modules with functionality obtained from the Gene Ontology (GO) database (25) as well as pathways acquired from the Reactome pathway database (26). The significance thresholds were set as 


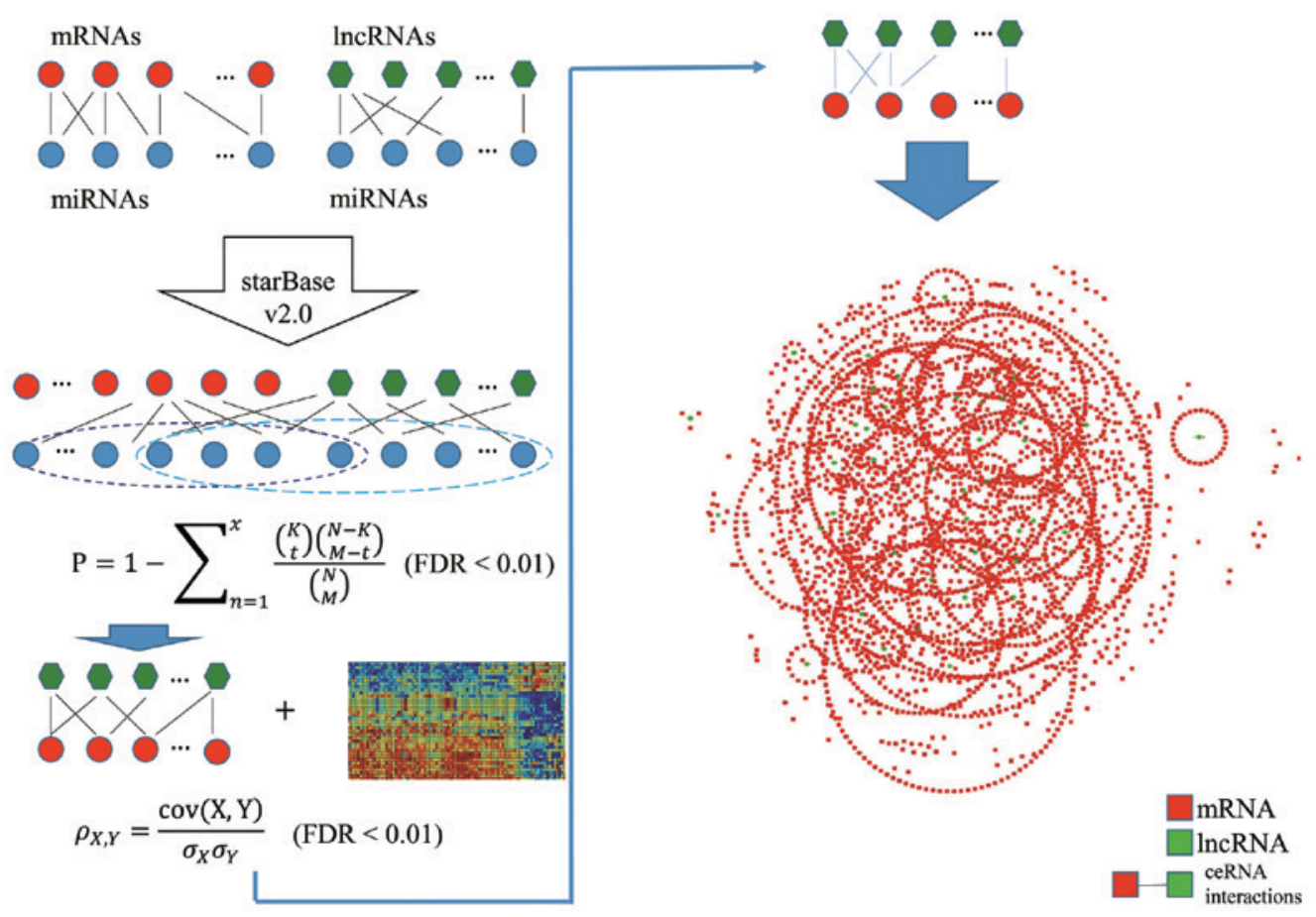

Figure 1. Identification flow chart of significantly co-expressed lncRNA-mRNA ceRNA pairs in CRC (left panel). LMCN sketch map (right panel). lncRNA, long non-coding RNA; ceRNA, competitive endogenous RNA; CRC, colorectal cancer; LMNC, IncRNA-mediated ceRNA network.

A

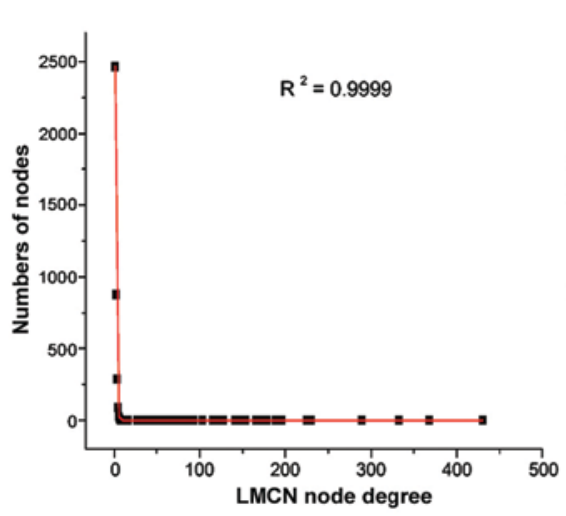

B

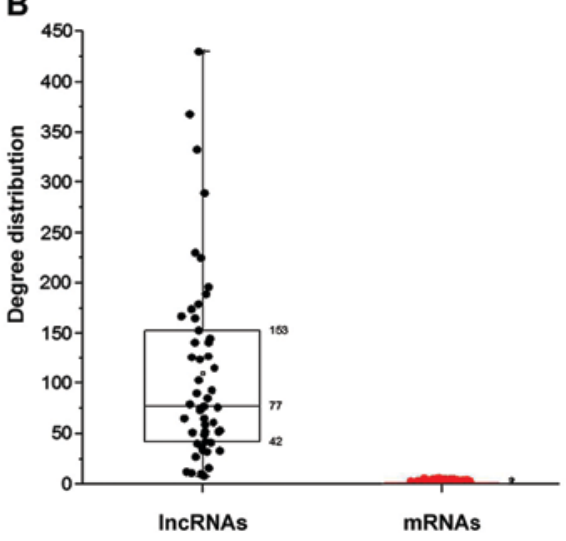

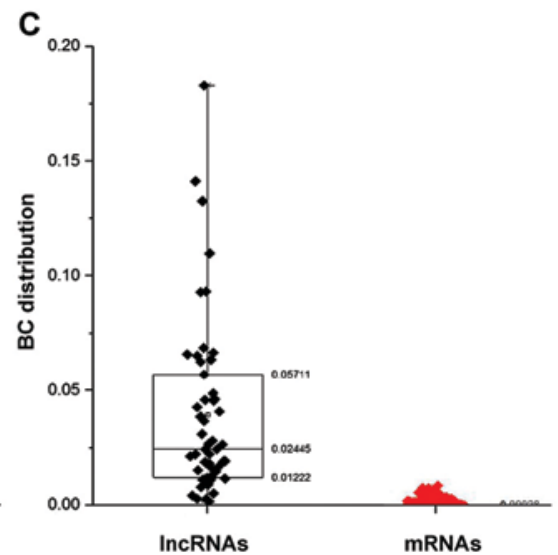

Figure 2. Topological analysis of LMCN. (A) The entire network reflected a power-law distribution. (B) The node degree distribution, and (C) the BC distribution of the lncRNAs were significantly higher than that of mRNAs. LMNC, lncRNA-mediated ceRNA network; BC, betweenness centrality; lncRNA, long non-coding RNA; ceRNA, competitive endogenous RNA.

$\mathrm{P}<0.01$ in functional analysis, and $\mathrm{P}<0.05$ in pathway analysis after Fisher's correction.

\section{Results}

The LMCN reveals specific topological properties. The LMCN was built with a multi-step method to assess the lncRNA-mediated ceRNA interactions. For miRNA-lncRNA interactions and miRNA-mRNA interactions in CRC, we collected and integrated full-scale datasets from the starBase v2.0 which provided experimentally supported high confidence miRNA-target interactions. A total of 265,728 pairs of miRNA-mRNA interactions as well as 598 pairs of lncRNA-miRNA interactions were acquired from a new expression profile covering a total of 8,522 genes consisting of 8,471 mRNAs and 51 lncRNAs. Based on the studies that lncRNA transcripts competed with endogenous mRNAs by binding to miRNAs that could be predicted with current miRNA target prediction approach $(27,28)$. The significance of common miRNAs shared by each lncRNA-mRNA pair was examined with a hypergeometric test. Then 51 lncRNAs, 8,125 mRNAs and 3,586 lncRNA-mRNA pairs were obtained. Pearson's correlation coefficient of the identified candidate ceRNA pair was calculated to confirm active ceRNA pairs in CRC. Eventually, we constructed and graphically simulated the LMCNs using the significantly co-expressed lncRNA-mRNA ceRNA pairs (Fig. 1). There were 51 lncRNAs, 3,752 mRNAs, and 5,617 ceRNA interactions in the LMCN. The statistics of nodes degree $\left(\mathrm{R}^{2}=0.9999\right)$ showed power law distributions (Fig. 2A), indicating that the 


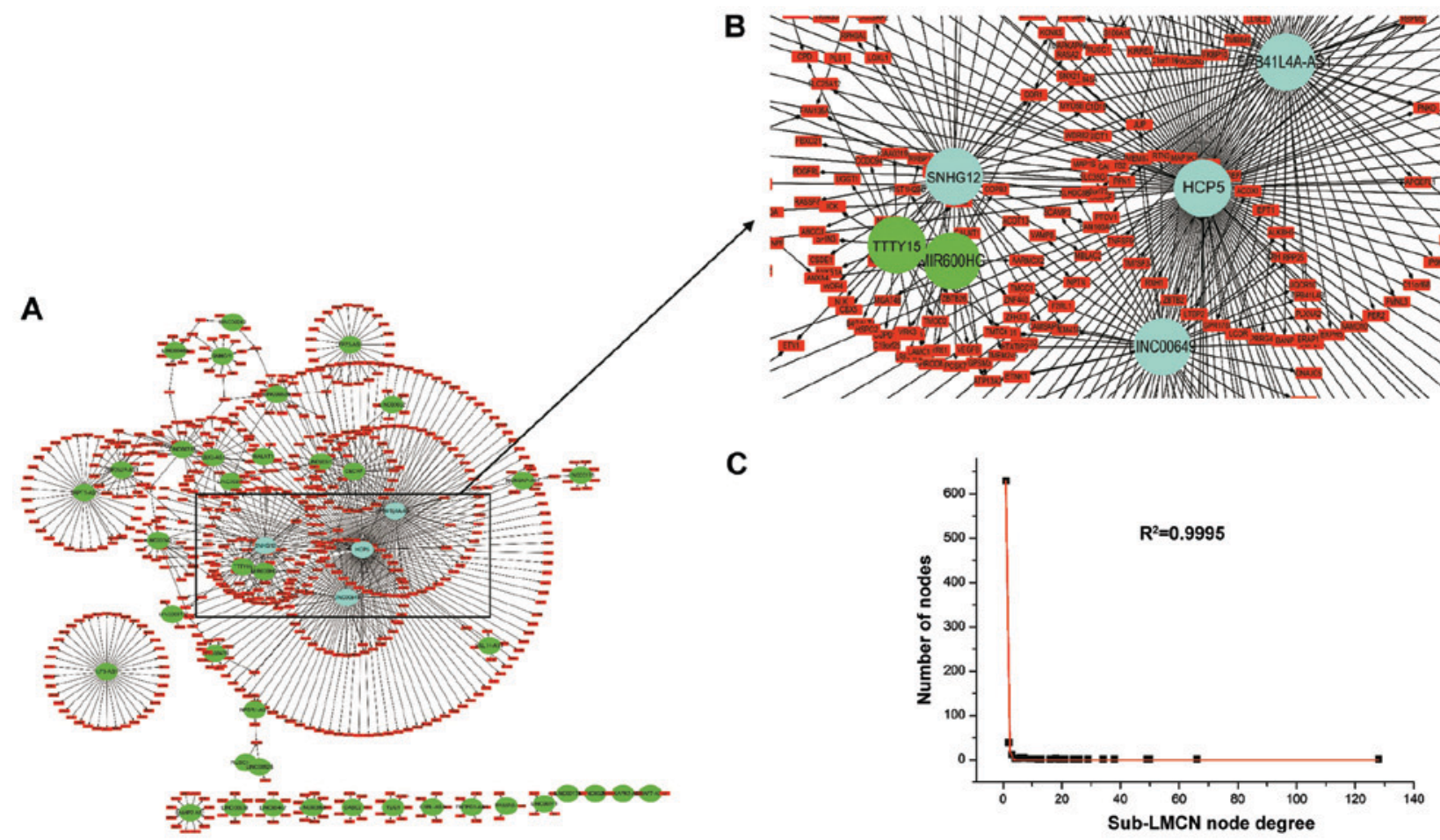

Figure 3. Overview of the highly competitive sub-network with Pearson's correlation coefficient deference $>0.5$. (A) The sub-LMCN contains 42 lncRNAs, 681 mRNAs, and 730 ceRNA interactions. The hub lncRNAs are shown as blue nodes, the other lncRNAs are shown as green nodes and mRNAs are shown as red nodes. (B) Amplified landscape map of the hub and bottleneck nodes. (C) The sub-LMCN had a scale-free structure complying with power-law distribution. LMNC, IncRNA-mediated ceRNA network; lncRNA, long non-coding RNA; ceRNA, competitive endogenous RNA.

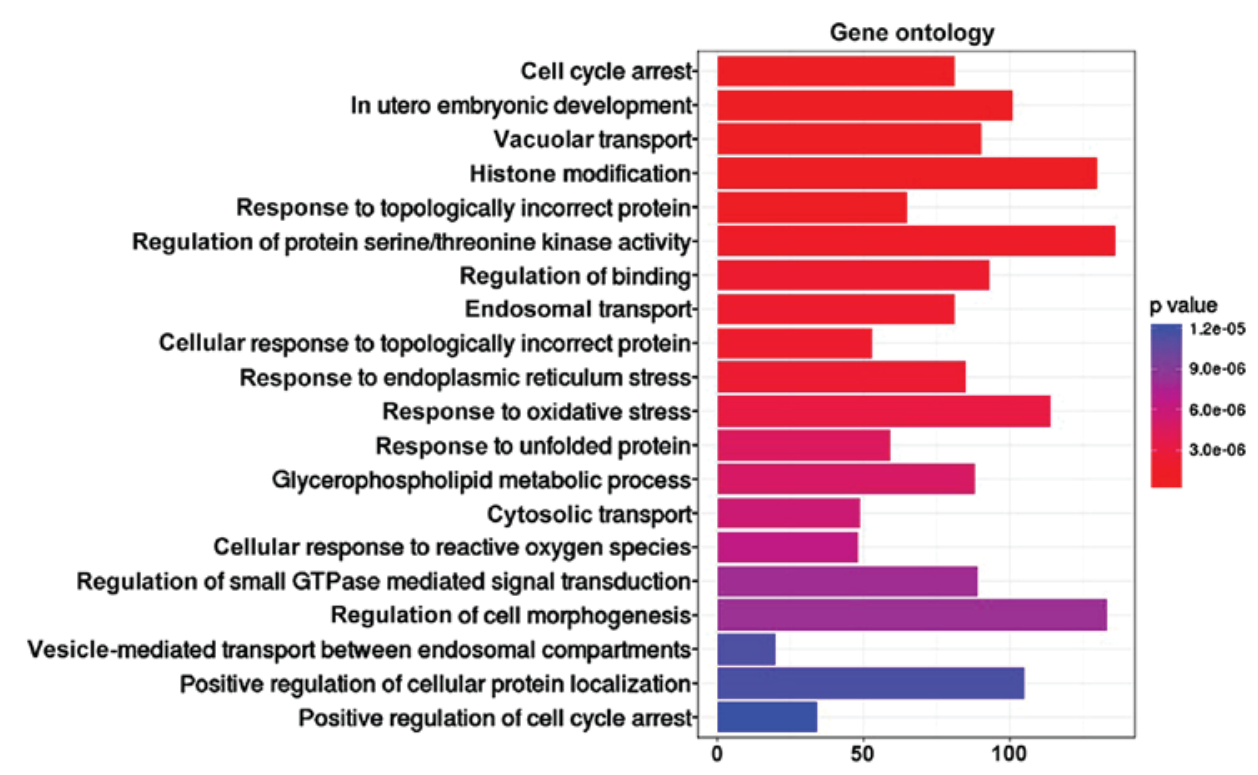

Figure 4. The top 20 enriched functions of mRNAs associated with lncRNAs in GO database. IncRNA, long non-coding RNA; GO, Gene Ontology

LMCN related to CRC is a scale-free network. Moreover, the lncRNA nodes exhibited more specific topological properties with more degrees and BCs in comparison with mRNA nodes (Fig. 2B and C).

HCP5, EPB41L4A-AS1, SNHG12, and LINC00649 are the hub lncRNAs in CRC. To determine the hub lncRNAs with highly specific properties in CRC, a sub-LMCN covering lncRNA-mRNA co-expression interactions with Pearson's correlation coefficient deference $>0.5$ between tumor and control groups was constructed (29). Results showed that the sub-LMCN also had a power-law distribution scaleless structure (Fig. 3C; $\mathrm{R}^{2}=0.9995$ ). The landscape map contained 42 lncRNAs, 681 mRNAs, and 730 ceRNA interactions (Fig. 3A). We found that HCP5, EPB41L4A-AS1, SNHG12, and LINC00649 had more node degrees and BCs in the sub-LMCN than others (Fig. 3B), indicating that they may be the prognostic markers in CRC. 
Table I. The top 20 enriched Reactome pathways of mRNAs associated with lncRNAs.

\begin{tabular}{|c|c|c|c|}
\hline No. & Reactome pathway & P-value (FDR) & No. of genes \\
\hline 1 & GPCR downstream signaling & $2.64 \mathrm{E}-29$ & 76 \\
\hline 2 & Signaling by GPCR & $4.09 \mathrm{E}-25$ & 93 \\
\hline 3 & GPCR ligand binding & $6.93 \mathrm{E}-13$ & 33 \\
\hline 4 & Class A/1 (Rhodopsin-like receptors) & $1.31 \mathrm{E}-09$ & 22 \\
\hline 5 & Signaling by Rho GTPases & $1.01 \mathrm{E}-06$ & 125 \\
\hline 6 & Peptide ligand-binding receptors & $1.05 \mathrm{E}-06$ & 11 \\
\hline 7 & Complement cascade & 7.60E-06 & 1 \\
\hline 8 & Axon guidance & $2.07 \mathrm{E}-05$ & 110 \\
\hline 9 & Developmental Biology & $5.78 \mathrm{E}-05$ & 158 \\
\hline 10 & G $\alpha$ (i) signalling events & $5.78 \mathrm{E}-05$ & 21 \\
\hline 11 & Initial triggering of complement & 3.70E-04 & 1 \\
\hline 12 & Membrane trafficking & $3.70 \mathrm{E}-04$ & 70 \\
\hline 13 & RHO GTPase effectors & $3.70 \mathrm{E}-04$ & 85 \\
\hline 14 & Asparagine $N$-linked glycosylation & $1.12 \mathrm{E}-03$ & 46 \\
\hline 15 & UPR & $1.12 \mathrm{E}-03$ & 37 \\
\hline 16 & Sema4D in semaphorin signaling & $1.66 \mathrm{E}-03$ & 16 \\
\hline 17 & Biological oxidations & $2.43 \mathrm{E}-03$ & 16 \\
\hline 18 & Scavenging heme from plasma & $2.43 \mathrm{E}-03$ & 1 \\
\hline 19 & Immunoregulatory interactions between a lymphoid and a non-lymphoid cell & $2.47 \mathrm{E}-03$ & 9 \\
\hline 20 & Translocation of GLUT4 to the plasma membrane & $2.85 \mathrm{E}-03$ & 27 \\
\hline
\end{tabular}

lncRNA, long non-coding RNA; FDR, false discovery rate; UPR, unfolded protein response.

Sixty-three functions and 66 pathways enriched from $G O$ and Reactome pathway database. To further validate the potential functional implication of lncRNAs, function and pathway enrichment analysis of mRNAs in the sub-LMCN were conducted based on GO and Reactome pathway database. The mRNAs associated with hub lncRNAs were obviously enriched in 63 GO terms, especially involved in cell cycle arrest, vacuolar transport, histone modification, and regulation of protein serine/threonine kinase activity (Fig. 4), and 66 Reactome pathways, including GPCR pathway, signaling by Rho GTPases, axon guidance and developmental biology (Table I).

\section{Discussion}

Considering the characteristics with hidden tumorigenesis, apt to local invasion and metastasis of CRC, what we primarily do for good prognosis is to ascertain pathogenesis, make early diagnosis and targeted individualized treatment. Bioinformatics is one of the interdisciplinaries involved in many fields, and one of its core elements is to study the internal expression regulatory mechanisms of genes and reveal essential laws of human diseases. lncRNA is an important type of gene expression regulatory factor in bioinformatics, and is involved in many human diseases, such as tumors (30), cardiovascular disease (31), inflammation (32) and autoimmune disease (33). The interconnections of ceRNAs play a crucial role in the development and physiology of tumors $(34,35)$. In the present study, we constructed a functional LMCN to explore the intermodulation relationship between lncRNAs and mRNAs, and predicted the functions and pathways involved in lncRNAs across $80 \mathrm{CRC}$ samples. Firstly, the LMCN was constructed through integrating the expression profiles of genome-wide lncRNA/mRNA and comprehensive interactions of miRNA-target. The LMCN presented a scale-free network conforming to power-law distributions, and the lncRNA nodes exhibited more specific topological properties with more degrees and BCs than mRNA nodes. Thus, these results indicated that LMCN has similar properties with many biological networks, and can be greatly organized into a structured rather than random network through a set of core lncRNA-mRNA competing principles $(36,37)$. Then a sub-LMCN was established to identify the hub and bottleneck lncRNAs with a threshold of Pearson's correlation coefficient deference $>0.5$. We found that HCP5, EPB41L4A-AS1, SNHG12, and LINC00649 have more node degrees and BCs in the landscape map. Finally, function enrichment analyses of mature mRNAs from GO and Reactome pathway databases were carried out to predict the functions of the obviously relative lncRNAs based on the GBA principle. As a result, the lncRNAs were found to be involved in 63 GO terms and 66 Reactome pathways, including the biological process of cell cycle arrest, vacuolar transport, histone modification, regulation of protein serine/threonine kinase activity, and the pathways of signaling by Rho GTPases, GPCR pathway, axon guidance, developmental biology had lower P-values and covered more mRNAs.

lncRNAs regulate the expression level of protein-coding genes in different ways, such as basic regulation process of genes and post-transcriptional regulation (38-40). In this study, we found that HCP5, EPB41L4A-AS1, SNHG12, and LINC00649 present stronger centrality and bridge-joint, 
meaning that they are more important than other lncRNAs in the onset and progress of CRC. Some investigations have shown that SNHG12 could increase cell cycle-related protein expression and suppress caspase-3 expression in CRC and human osteosarcoma cells. Moreover, silencing SNHG12 expression can inhibit triple-negative breast cancer cell proliferation, and it has been found that SNHG12, as an endogenous sponge of miR-199a/b-5p, regulates MLK3 expression in hepatocellular carcinoma and affects the activation of the NF- $\mathrm{\kappa B}$ pathway (41-44). Therefore, SNHG12 may be considered as a potential biomarker and a promising therapeutic target for CRC and other cancers. It has been reported that (HCP5) is highly expressed in glioma tissues and U87 and U251 cells, which can promote the malignant biological behavior of glioma cells by enhancing proliferation, migration as well as invasion, and inhibiting apoptosis (45). Furthermore, a single nucleotide polymorphism in HCP5 (rs2244546) has been confirmed as a strong predictor of hepatitis $\mathrm{C}$ virus-related hepatocellular carcinoma in the Swiss Hepatitis C Cohort Study (46). EPB41L4A-AS1 was found a risky lncRNA in ovarian cancer (47). HCP5 and EPB41L4A-AS1 could be boldly inferred as potential lncRNA biomarkers for the diagnosis, evaluation and gene-targeted therapy of CRC, but further studies needs to be carried out to verify this inference.

$\mathrm{GO}$ analysis suggested that the target genes of hub lncRNAs are significantly associated with cell cycle and histone modification. Cell cycle regulation is of great significance in maintaining homeostasis and preventing cancer, and its abnormity has been long regarded as an important intermediate link for the tumor development (48-50). The basic function of histone modification is to regulate gene expression, and phosphorylation of histones is not only an important intermediate step of certain signal transduction pathways, but also affects cell division and cycle through combining with other types of modification $(51,52)$. Therefore, the result in this study was consistent with previous reports, and the occurrence and development of CRC are closely related to cell cycle and histones modification. The reactome pathway analysis showed that the lncRNAs might participate in the Rho GTPases and axon guidance pathways. Rho GTPases family is an intracellular signal transducer that connects cell surface signals to multiple intracellular reactions. They playe a critical role in various cellular processes, including cell morphology, gene transcription, cell cycle progression, and cell adhesion $(53,54)$. Furthermore, it has been reported that oncogene-specific cell migration and invasion pathways are mediated by Rho GTPases in colon cancer cells (55). Semaphorins primarily known as ligands for plexins and neuropilins are key members of axon guidance molecules (56). Many studies have found that semaphorin proteins have certain modulatory effects on tumorous occurrence and development (57-59), for example, the axon guidance molecule semaphorin $3 \mathrm{~F}$ was found in normal neuroendocrine cells, while it was lost in most human primary tumors and all metastases. Axon guidance molecule semaphorin $3 \mathrm{~F}$ plays a negative regulator role in the development of tumor in ileal neuroendocrine tumors (60). Additionally, the inhibition of migration, invasion and epithelial to mesenchymal transition has been revealed because of the overexpression of SEMA3A by suppressing the expression of NF-kB and SNAI2 in head and neck squamous cell carcinoma (HNSCC); whereas, a shorter overall survival and more important independent prognostic significance could be found in HNSCC patients with lower SEMA3A expression (61). Therefore, axon guidance cues could be promising targets for personalized anticancer therapies. In short, the results of the present study indicated that cancer-related LMCN contributes in improving the identification of biomarkers and promotes the development of CRC therapy.

In conclusion, we have provided the LMCN landscape across 80 CRC samples with a new method. The specific topological properties and synergistic competition effects of IncRNAs may reveal the regulatory interactions of coding mRNAs in CRC.

\section{Acknowledgements}

Not applicable.

\section{Funding}

No funding was received.

\section{Availability of data and materials}

The datasets used and/or analyzed during the present study are available from the corresponding author on reasonable request.

\section{Authors' contributions}

$\mathrm{MH}$ analyzed the data and was also a major contributor in writing the manuscript. YL and YX made substantial contribution to the conception and design of the study. MH, YL performed statistical analysis. YX was involved in the drafting of the manuscript and gave the final approval for publication. All authors read and approved the final manuscript.

\section{Ethics approval and consent to participate}

Not applicable.

\section{Patient consent for publication}

Not applicable.

\section{Competing interests}

The authors declare that they have no competing interests.

\section{References}

1. Torre LA, Bray F, Siegel RL, Ferlay J, Lortet-Tieulent J and Jemal A: Global cancer statistics, 2012. CA Cancer J Clin 65: 87-108, 2015.

2. Marley AR and Nan H: Epidemiology of colorectal cancer. Int J Mol Epidemiol Genet 7: 105-114, 2016.

3. Bretthauer M: Colorectal cancer screening. J Intern Med 270: 87-98, 2011.

4. Itzel T, Scholz P, Maass T, Krupp M, Marquardt JU, Strand S, Becker D, Staib F, Binder H, Roessler S, et al: Translating bioinformatics in oncology: Guilt-by-profiling analysis and identification of KIF18B and CDCA3 as novel driver genes in carcinogenesis. Bioinformatics 31: 216-224, 2015.

5 . Fortney $\mathrm{K}$ and Jurisica I: Integrative computational biology for cancer research. Hum Genet 130: 465-481, 2011. 
6. Hao L, Leng J, Xiao R, Kingsley T, Li X, Tu Z, Yang X, Deng X, Xiong $\mathrm{M}$, Xiong J, et al: Bioinformatics analysis of the prognostic value of Tripartite Motif 28 in breast cancer. Oncol Lett 13: 2670-2678, 2017

7. Fritah S, Niclou SP and Azuaje F: Databases for lncRNAs: A comparative evaluation of emerging tools. RNA 20: 1655-1665, 2014

8. Nagano T and Fraser P: No-nonsense functions for long noncoding RNAs. Cell 145: 178-181, 2011.

9. Dong Y, Huo X, Sun R, Liu Z, Huang M and Yang S: LncRNA Gm15290 promotes cell proliferation and invasion in non-small cell lung cancer through directly interacting with and suppressing the tumor suppressor miR-615-5p. Oncol Res: May 5, 2017 (Epub ahead of print)

10. Wen Q, Liu Y, Lyu H, Xu X, Wu Q, Liu N, Yin Q, Li J and Sheng X: Long noncoding RNA GAS5, which acts as a tumor suppressor via microRNA 21, regulates cisplatin resistance expression in cervical cancer. Int J Gynecol Cancer 27: 1096-1108, 2017.

11. Hou Z, Xu X, Fu X, Tao S, Zhou J, Liu S and Tan D: HBx-related long non-coding RNA MALAT1 promotes cell metastasis via up-regulating LTBP3 in hepatocellular carcinoma. Am J Cancer Res 7: 845-856, 2017.

12. Huan J, Xing L, Lin Q, Xui H and Qin X: Long noncoding RNA CRNDE activates Wnt/ $\beta$-catenin signaling pathway through acting as a molecular sponge of microRNA-136 in human breast cancer. Am J Transl Res 9: 1977-1989, 2017.

13. Ebert MS and Sharp PA: Emerging roles for natural microRNA sponges. Curr Biol 20: R858-R861, 2010.

14. Cesana M, Cacchiarelli D, Legnini I, Santini T, Sthandier O, Chinappi M, Tramontano A and Bozzoni I: A long noncoding RNA controls muscle differentiation by functioning as a competing endogenous RNA. Cell 147: 358-369, 2011

15. Sumazin $P$, Yang $X$, Chiu HS, Chung WJ, Iyer A, Llobet-Navas D, Rajbhandari P, Bansal M, Guarnieri P, Silva J, et al: An extensive microRNA-mediated network of RNA-RNA interactions regulates established oncogenic pathways in glioblastoma. Cell 147: 370-381, 2011.

16. Wang H, Niu L, Jiang S, Zhai J, Wang P, Kong F and Jin X: Comprehensive analysis of aberrantly expressed profiles of lncRNAs and miRNAs with associated ceRNA network in muscle-invasive bladder cancer. Oncotarget 7: 86174-86185, 2016

17. Loo LW, Cheng I, Tiirikainen M, Lum-Jones A, Seifried A, Dunklee LM, Church JM, Gryfe R, Weisenberger DJ, Haile RW, et al: cis-Expression QTL analysis of established colorectal cancer risk variants in colon tumors and adjacent normal tissue. PLoS One 7: e30477, 2012.

18. Li JH, Liu S, Zhou H, Qu LH and Yang JH: starBase v2.0 Decoding miRNA-ceRNA, miRNA-ncRNA and protein-RNA interaction networks from large-scale CLIP-Seq data. Nucleic Acids Res 42 (D1): D92-D97, 2014.

19. Mukaka MM: Statistics corner: A guide to appropriate use of correlation coefficient in medical research. Malawi Med J 24: 69-71, 2012.

20. Ravasz E, Somera AL, Mongru DA, Oltvai ZN and Barabási AL: Hierarchical organization of modularity in metabolic networks. Science 297: 1551-1555, 2002.

21. Chen X: Prediction of optimal gene functions for osteosarcoma using network-based-guilt by association method based on gene oncology and microarray profile. J Bone Oncol 7: 18-22, 2017.

22. Peña-Castillo L, Tasan M, Myers CL, Lee H, Joshi T, Zhang C, Guan Y, Leone M, Pagnani A, Kim WK, et al: A critical assessment of Mus musculus gene function prediction using integrated genomic evidence. Genome Biol 9 (Suppl 1): S2, 2008

23. Pavlidis P and Gillis J: Progress and challenges in the computational prediction of gene function using networks: 2012-2013 update. F1000Res 2: 230, 2013

24. Kominakis A, Hager-Theodorides AL, Zoidis E, Saridaki A, Antonakos G and Tsiamis G: Combined GWAS and 'guilt by association'-based prioritization analysis identifies functional candidate genes for body size in sheep. Genet Sel Evol 49: 41, 2017.

25. Huntley RP, Sawford T, Mutowo-Meullenet P, Shypitsyna A, Bonilla C, Martin MJ and O'Donovan C: The GOA database: Gene Ontology annotation updates for 2015. Nucleic Acids Res 43 (D1): D1057-D1063, 2015.

26. Haw R and Stein L: Using the reactome database. Curr Protoc Bioinform 38: 1-23, 2012

27. Zou T, Jaladanki SK, Liu L, Xiao L, Chung HK, Wang JY, Xu Y, Gorospe $\mathrm{M}$ and Wang JY: H19 long noncoding RNA regulates intestinal epithelial barrier function via microRNA 675 by interacting with RNA-binding protein HuR. Mol Cell Biol 36: 1332-1341, 2016
28. Dey BK, Pfeifer K and Dutta A: The H19 long noncoding RNA gives rise to microRNAs miR-675-3p and miR-675-5p to promote skeletal muscle differentiation and regeneration. Genes Dev 28: 491-501, 2014

29. Wang P, Ning S, Zhang Y, Li R, Ye J, Zhao Z, Zhi H, Wang T, Guo Z and Li X: Identification of lncRNA-associated competing triplets reveals global patterns and prognostic markers for cancer. Nucleic Acids Res 43: 3478-3489, 2015.

30. Misawa A, Takayama K, Urano T and Inoue S: Androgen-induced long noncoding RNA (IncRNA) SOCS2-AS1 promotes cell growth and inhibits apoptosis in prostate cancer cells. J Biol Chem 291: 17861-17880, 2016.

31. Shi Q and Yang X: Circulating microRNA and long noncoding RNA as biomarkers of cardiovascular diseases. J Cell Physiol 231: 751-755, 2016.

32. Su S, Liu J, He K, Zhang M, Feng C, Peng F, Li B and Xia X: Overexpression of the long noncoding RNA TUG1 protects against cold-induced injury of mouse livers by inhibiting apoptosis and inflammation. FEBS J 283: 1261-1274, 2016.

33. Wu GC, Pan HF, Leng RX, Wang DG, Li XP, Li XM and Ye DQ: Emerging role of long noncoding RNAs in autoimmune diseases. Autoimmun Rev 14: 798-805, 2015.

34. Zhang Y, Xu Y, Feng L, Li F, Sun Z, Wu T, Shi X, Li J and Li X: Comprehensive characterization of $\operatorname{lncRNA}-m R N A$ related ceRNA network across 12 major cancers. Oncotarget 7: 64148-64167, 2016.

35. Chiu YC, Wang LJ, Lu TP, Hsiao TH, Chuang EY and Chen Y: Differential correlation analysis of glioblastoma reveals immune ceRNA interactions predictive of patient survival. BMC Bioinformatics 18: 132, 2017.

36. Rider AK, Milenković T, Siwo GH, Pinapati RS, Emrich SJ, Ferdig MT and Chawla NV: Networks' characteristics matter for systems biology. Netw Sci (Camb Univ Press) 2: 139-161, 2014.

37. Wang P, Lü J and Yu X: Identification of important nodes in directed biological networks: A network motif approach. PLoS One 9: e106132, 2014.

38. Xing Z, Lin A, Li C, Liang K, Wang S, Liu Y, Park PK, Qin L, Wei Y, Hawke DH, et al: lncRNA directs cooperative epigenetic regulation downstream of chemokine signals. Cell 159: $1110-1125,2014$

39. Roberts TC, Morris KV and Weinberg MS: Perspectives on the mechanism of transcriptional regulation by long non-coding RNAs. Epigenetics 9: 13-20, 2014

40. Shi X, Sun M, Wu Y, Yao Y, Liu H, Wu G, Yuan D and Song Y: Post-transcriptional regulation of long noncoding RNAs in cancer. Tumour Biol 36: 503-513, 2015.

41. Wang JZ, Xu CL, Wu H and Shen SJ: LncRNA SNHG12 promotes cell growth and inhibits cell apoptosis in colorectal cancer cells. Braz J Med Biol Res 50: e6079, 2017.

42. Wang O, Yang F, Liu Y, Lv L, Ma R, Chen C, Wang J, Tan Q, Cheng Y, Xia E, et al: C-MYC-induced upregulation of lncRNA SNHG12 regulates cell proliferation, apoptosis and migration in triple-negative breast cancer. Am J Transl Res 9: 533-545, 2017.

43. Lan T, Ma W, Hong Z, Wu L, Chen X and Yuan Y: Long non-coding RNA small nucleolar RNA host gene 12 (SNHG12) promotes tumorigenesis and metastasis by targeting miR-199a/b-5p in hepatocellular carcinoma. J Exp Clin Cancer Res 36: 11, 2017.

44. Ruan W, Wang P, Feng S, Xue Y and Li Y: Long non-coding RNA small nucleolar RNA host gene 12 (SNHG12) promotes cell proliferation and migration by upregulating angiomotin gene expression in human osteosarcoma cells. Tumour Biol 37: 4065-4073, 2016.

45. Teng H, Wang P, Xue Y, Liu X, Ma J, Cai H, Xi Z, Li Z and Liu Y: Role of HCP5-miR-139-RUNX1 feedback loop in regulating malignant behavior of glioma cells. Mol Ther 24: 1806-1822, 2016.

46. Lange CM, Bibert S, Dufour JF, Cellerai C, Cerny A, Heim MH, Kaiser L, Malinverni R, Müllhaupt B, Negro F, et al; Swiss Hepatitis C Cohort Study Group: Comparative genetic analyses point to HCP5 as susceptibility locus for $\mathrm{HCV}$-associated hepatocellular carcinoma. J Hepatol 59: 504-509, 2013.

47. Zhou M, Wang X, Shi H, Cheng L, Wang Z, Zhao H, Yang L and Sun J: Characterization of long non-coding RNA-associated ceRNA network to reveal potential prognostic lncRNA biomarkers in human ovarian cancer. Oncotarget 7: 12598-12611, 2016.

48. Xiao H, Xiao W, Cao J, Li H, Guan W, Guo X, Chen K, Zheng T, Ye Z, Wang J, et al: miR-206 functions as a novel cell cycle regulator and tumor suppressor in clear-cell renal cell carcinoma. Cancer Lett 374: 107-116, 2016 
49. Chukkapalli S, Amessou M, Dekhil H, Dilly AK, Liu Q Bandyopadhyay S, Thomas RD, Bejna A, Batist G and Kandouz M: Ehd3, a regulator of vesicular trafficking, is silenced in gliomas and functions as a tumor suppressor by controlling cell cycle arrest and apoptosis. Carcinogenesis 35: 877-885, 2014.

50. Ouyang Q, Xu L, Cui H, Xu M and Yi L: MicroRNAs and cell cycle of malignant glioma. Int J Neurosci 126: 1-9, 2016.

51. Banerjee T and Chakravarti D: A peek into the complex realm of histone phosphorylation. Mol Cell Biol 31: 4858-4873, 2011.

52. Kelly AE, Ghenoiu C, Xue JZ, Zierhut C, Kimura $\mathrm{H}$ and Funabiki H: Survivin reads phosphorylated histone $\mathrm{H} 3$ threonine 3 to activate the mitotic kinase Aurora B. Science 330: 235-239, 2010.

53. Zegers MM and Friedl P: Rho GTPases in collective cell migration. Small GTPases 5: e28997, 2014.

54. Lin Y and Zheng Y: Approaches of targeting Rho GTPases in cancer drug discovery. Expert Opin Drug Discov 10: 991-1010, 2015.

55. Makrodouli E, Oikonomou E, Koc M, Andera L, Sasazuki T, Shirasawa $S$ and Pintzas A: BRAF and RAS oncogenes regulate Rho GTPase pathways to mediate migration and invasion properties in human colon cancer cells: A comparative study. Mol Cancer 10: 118, 2011.

56. Andermatt I, Wilson NH, Bergmann T, Mauti O, Gesemann M, Sockanathan $\mathrm{S}$ and Stoeckli ET: Semaphorin 6B acts as a receptor in post-crossing commissural axon guidance. Development 141: 3709-3720, 2014.
57. Ge C, Li Q, Wang $\mathrm{L}$ and $\mathrm{Xu} \mathrm{X}$ : The role of axon guidance factor semaphorin $6 \mathrm{~B}$ in the invasion and metastasis of gastric cancer. J Int Med Res 41: 284-292, 2013.

58. Mumblat Y, Kessler O, Ilan N and Neufeld G: Full-length semaphorin-3C is an inhibitor of tumor lymphangiogenesis and metastasis. Cancer Res 75: 2177-2186, 2015.

59. Gu C and Giraudo E: The role of semaphorins and their receptors in vascular development and cancer. Exp Cell Res 319: 1306-1316, 2013.

60. Bollard J, Massoma P, Vercherat C, Blanc M, Lepinasse F, Gadot N, Couderc C, Poncet G, Walter T, Joly MO, et al: The axon guidance molecule semaphorin $3 \mathrm{~F}$ is a negative regulator of tumor progression and proliferation in ileal neuroendocrine tumors. Oncotarget 6: 36731-36745, 2015.

61. Wang Z, Chen J, Zhang W, Zheng Y, Wang Z, Liu L, Wu H, Ye J, Zhang W, Qi B, et al: Axon guidance molecule semaphorin 3A is a novel tumor suppressor in head and neck squamous cell carcinoma. Oncotarget 7: 6048-6062, 2016.

This work is licensed under a Creative Commons Attribution-NonCommercial-NoDerivatives 4.0 International (CC BY-NC-ND 4.0) License. 